\title{
Tribological properties of novel palygorskite nanoplatelets used as oil-based lubricant additives
}

\author{
Kunpeng WANG ${ }^{1,2}$, Huaichao WU ${ }^{1, *}$, Hongdong WANG ${ }^{2}$, Yuhong LIU $^{2, *}$, Lv YANG ${ }^{1}$, Limei ZHAO ${ }^{1}$ \\ ${ }^{1}$ School of Mechanical Engineering, Guizhou University, Guiyang 550025, China \\ ${ }^{2}$ State Key Laboratory of Tribology, Tsinghua University, Beijing 100084, China \\ Received: 19 July 2019 / Revised: 24 October 2019 / Accepted: 03 December 2019 \\ (C) The author(s) 2019.
}

\begin{abstract}
Layered palygorskite (PAL), commonly called attapulgite, is a natural inorganic clay mineral composed of magnesium silicate. In this study, an aqueous miscible organic solvent treatment method is adopted to prepare molybdenum-dotted palygorskite (Amo-PMo) nanoplatelets, which greatly improved the specific surface area of PAL and the dispersion effect in an oil-based lubricant system. Their layered structure and size were confirmed using transmission electron microscopy (TEM) and atomic force microscopy. Following a tribological test lubricated with three additives (PAL, organic molybdenum (SN-Mo), and Amo-PMo), it was found that the sample of $0.5 \mathrm{wt} \%$ Amo-PMo exhibited the best tribological properties with a coefficient of friction of 0.09 . Moreover, the resulting wear scar diameter and wear volume of the sliding ball surface were $63 \%$ and $49.6 \%$ of those lubricated with base oil, respectively. Its excellent lubricating performance and self-repairing ability were mainly attributed to the generated $\mathrm{MoS}_{2}$ adsorbed on the contact surfaces during the tribochemical reaction, thereby effectively preventing the direct collision between asperities on sliding solid surfaces. Thus, as-prepared Amo-PMo nanoplatelets show great potential as oil-based lubricant additives, and this study enriches the existing application of PAL in industry.
\end{abstract}

Keywords: palygorskite (PAL); layered material; lubricant additive; wear; tribofilm

\section{Introduction}

Industries have been constantly seeking environmentally friendly and economical lubricants in an attempt to reduce energy waste caused by friction. In the past few years, nanomaterials (e.g., multilayer graphene [1] and two-dimensional layered molybdenum disulphide [2]) have been widely dispersed in lubricants because of their relatively weak intermolecular interactions [3] (i.e., van der Waals interactions) and the formation of a lubricating oxide layer on the worn surface to improve the anti-wear performance of sliding solid surfaces [4].

Recently, mineral clays have attracted considerable interest for use as lubricant additives due to their affordability and availability. Layered palygorskite
(PAL) possesses an ideal layered structure with a twodimensional (2D) flake morphology. The basic formula of PAL can be expressed as $\mathrm{Mg}_{2}\left(\mathrm{H}_{2} \mathrm{O}\right)_{4}\left(\mathrm{Si}_{4} \mathrm{O}_{10}\right)_{2}(\mathrm{OH})_{2}$. Because of its high adsorbing property and unique interlaminar structures, PAL has been widely investigated in fields such as catalysts [5], functionalized film [6], and nanocomposites [7], and PAL can be used for wastewater treatment [8] and pollutant adsorption [9] applications. SN-Mo facilitates passivation and generates a more stable passivating oxide film on sliding surfaces [10]. However, SN-Mo does not disperse well in an organic solvent, which limits its tribological application as a lubricant additive. The aqueous miscible organic solvent treatment (AMOST) method enables nano-sized composite materials to be stably dispersed in organic solvents [11]. Some natural

* Corresponding authors: Huaichao WU, E-mail: hcwu@gzu.edu.cn; Yuhong LIU, E-mail: liuyuhong@tsinghua.edu.cn 
mineral powders containing silicates, such as serpentine $[12,13]$ and PAL [14], can be used as self-lubricating materials and added to lubricant oils to form a selfrepairing film with a certain thickness on the metal sliding surfaces. This self-repairing film is a hard and protective film formed by oxide [15]. PAL is currently used in drilling fluids to assist in removing cutting debris from the wellbore and to maintain debris dispersion [16]. Compound PAL and polytetrafluoroethylene (PEFE) were tested on a block-on-ring tribotester. The tribological properties of the heat treated and acid treated PAL were found to be superior to those of the untreated PAL. Filling the treated nano-palygorskite into PTFE make it possible to promote the formation of tribofilm on the surface $[17,18]$. Nan et al. [19, 20] applied $\mathrm{Cu}$ and $\mathrm{Ni}$ nanoparticles to improve the tribological properties of based oil. A smooth tribofilm mainly composed of metal elemental and metal oxide was formed on the sliding surface. Tian et al. [21] investigated the use of spent methyl-violet (MV)-loaded PAL as multifunctional nanoparticles, which were synthesized by a simple one-pot approach with the assistance of $\mathrm{AgNO}_{3}$. The results revealed that the $\mathrm{Ag}(\mathrm{I})$ ions promoted the carbonation of the MV molecules and led to the formation of nanosized composites with excellent adsorption properties. However, some disadvantages exist with the aforementioned PAL application. For example, the dispersion effect of the lubricant additive is rarely considered. If the dispersion effect is not satisfactory, the lubricant additive cannot be applied to a mechanical industry.

Here, we select the AMOST method to synthesize a new type of molybdenum-dotted PAL nanoplatelets. The as-synthesized samples, their morphologies and physicochemical characteristics are analyzed by transmission electron microscopy (TEM), scanning electron microscopy (SEM), atomic force microscopy (AFM), X-ray photoelectron spectroscopy (XPS), and the technology of dynamic light scattering (DLS). After Amo-PMo nanoplatelets are dispersed in the base oil, the relationship between surface functionalization and dispersion efficiency is investigated. Then, the friction and wear properties of lubricants with different Amo-PMo nanoplatelets are evaluated by a four-ball tribometer. Compared with synthetic additives and commercial additives, it is found that the Amo-PMo possesses a superior ability to increase the tribological properties of the lubricating fluid. The sliding contact surfaces are analyzed after the experiment to determine the composition of the tribofilm when the lubricant additive is added. Finally, based on these results, the tribological behavior is described and a potential lubrication mechanism of the nanoplatelets is proposed.

\section{Experimental}

\subsection{Materials preparation}

SN 2011 non-sulfur-phosphorus organic molybdenum (SN-Mo) and 3-triethoxysilylpropylamine (APTES) were ordered from Gelest, and pure PAL (95\%) was obtained from Dafang, Guizhou, China. The ULTRA-S $150 \mathrm{~N}$ base oil (Korea Sangyong brand), ethylene glycol, urea, acetone, anhydrous ethanol, and ethylbenzene were obtained from China National Pharmaceutical Co., Ltd. All the reagents were of analytical grade. Pure PAL was first suspended in $180 \mathrm{~mL}$ of a $\mathrm{HCl}(4 \mathrm{M})$ aqueous solution to achieve a concentration of $100 \mathrm{~g} / \mathrm{L}$ and the solution was mechanically stirred at $900 \mathrm{rpm}$ for $1 \mathrm{~h}$. In addition, the acid-activated PAL was washed with ultrapure water until neutralized and then dried under a vacuum at $90^{\circ} \mathrm{C}$. The acid-activated sample was next placed in a muffle furnace and thermally activated at $400{ }^{\circ} \mathrm{C}$ for $1 \mathrm{~h}$. The activated PAL was then formulated into a $20 \mathrm{~mL}$ aqueous solution (A) at a concentration of $40 \mathrm{~g} / \mathrm{L}$. Next, $2.8 \mathrm{mmol}$ of $\mathrm{CO}\left(\mathrm{NH}_{2}\right)_{2}$ and different amounts of SN-Mo and APTES were dispersed in $80 \mathrm{~mL}$ of an ethylene glycol solution (B), and solution A was poured into solution $B$ under nitrogen protection. A homogeneous mixed solution was obtained by sonication, and the aqueous mixture was transferred to a $100 \mathrm{~mL}$ teflon-lined autoclave and heated to $100{ }^{\circ} \mathrm{C}$ for $24 \mathrm{~h}$. Finally, the reaction slurry was collected by centrifugation, washed with acetone, dispersed in the base oil, and stirred at room temperature for $1 \mathrm{~h}$. The washing and dispersion process were repeated three times on the slurry. After washing, the slurry was dried in a vacuum oven for $24 \mathrm{~h}$.

\subsection{Tribological test and evaluation}

The base oil with Amo-PMo nanoplatelets was tested using a MS-10A four-ball tribometer (Xiamen Tenkey 
Automation Co., Ltd., China). The temperature was set at $75{ }^{\circ} \mathrm{C}$, the relative sliding speed was $1,200 \mathrm{r} / \mathrm{min}$, the normal load was $400 \mathrm{~N}$, and the running time of the test was $60 \mathrm{~min}$. The ball diameter $(\phi)$, elastic modulus, Poisson's ratio, and Rockwell hardness of GCr15 bearing steel were $12.7 \mathrm{~mm}, 210 \mathrm{GPa}, 0.3$, and 64.66 , respectively. The sample had a surface roughness of $R a 0.2$ and a relative humidity of $\sim 30 \%$. The base oil was a hydroisomerizing waxy synthetic oil. The flash point, pour point, density, and sulfur content of the base oil were $232,-17^{\circ} \mathrm{C}, 854.4 \mathrm{~kg} / \mathrm{m}^{3}$, and $<1 \mathrm{ug} / \mathrm{g}$, respectively. At the beginning of the test, the dynamic viscosity of the base oil was $29.5 \mathrm{cP}$ at room temperature. Following the tribological test, the dynamic viscosity of the base oil was $17.5 \mathrm{cP}$ at $75{ }^{\circ} \mathrm{C}$. The dynamic viscosity of the lubricating fluid was tested at shear rates of 10 to $1,000 \mathrm{~s}^{-1}$. During the test of each dynamic viscosity, the viscosity did not change significantly with the shear rate, indicating that the lubricating fluid was a Newtonian fluid. All the experiments were run three times to ensure repeatability. Prior to the experiment, the steel bearing balls were washed with ethanol, petroleum ether, and acetone. After the experiment, the samples were ultrasonically cleaned, and the wear scars were observed using a threedimensional (3D) white light interferometer (ZYGO NexView) and SEM (FEI QUANTA 200FEG). A Rigaku Miniflex X-ray diffractometer with a variable slit width was used to generate the $X$-ray diffraction patterns, and data were collected at a scanning rate of $2\left(^{\circ}\right) / \mathrm{min}$ from $2 \theta=5^{\circ}$ to $65^{\circ}$ with $\mathrm{Co} \mathrm{K} \alpha$ radiation $(\lambda=0.17902 \mathrm{~nm})$. The TEM images were obtained on a JEOL JSM2010 transmission electron microscope with an accelerating voltage of $200 \mathrm{kV}$. An MFP-3D-SA model AFM system was used to examine the surface topography of the nanoplatelets deposited on mica wafers. SEM and energy dispersive X-ray spectrometry (EDS) analyses were performed on an FEI QUANTA 200FEG scanning microscope with an accelerating voltage of $30 \mathrm{kV}$. The surface chemical composition was explored by XPS with a PHI Quantera II spectrometer equipped with a monochromated $\mathrm{Al} \mathrm{K \alpha}$ X-ray radiation source and a hemispherical electron analyzer. The XPS spectra were recorded in constant pass energy mode at a value of $100 \mathrm{eV}$, and all the binding energies were calibrated using the $\mathrm{C} 1 \mathrm{~s}$ peak at $284.6 \mathrm{eV}$ as the reference.

\section{Results and discussion}

\subsection{Chemical and structural properties of the Amo- PMo nanoplatelets}

The AMOST method was used to synthesize AmoPMo. To confirm the crystal structure of PAL at different processing stages, we performed XRD measurements. In Fig. 1(a), the diffraction peaks of PAL were clearly similar to the typical diffraction peaks reported by Zhang et al. [22]. The diffraction peaks observed for the Amo-PMo sample were at $2 \theta=23.3^{\circ}, 27.3^{\circ}$, and $39.1^{\circ}$, which were caused by $\mathrm{MoO}_{3}$, indicating that the synthesized material contained molybdenum. Because the characteristic peak of PAL did not change, the PAL structure was not damaged [23]. Figure S1 in the Electronic Supporting Material (ESM) shows that the acid activation treatment and thermal activation treatment cannot change the characteristic peak of PAL, which effectively explain the high corrosion resistance and good thermal stability of PAL. In addition, the difference between the synthesized Amo-PMo and PAL samples, as studied through Fourier transform infrared (IR) spectroscopy, is displayed in Fig. 1(b). The main difference between the two IR
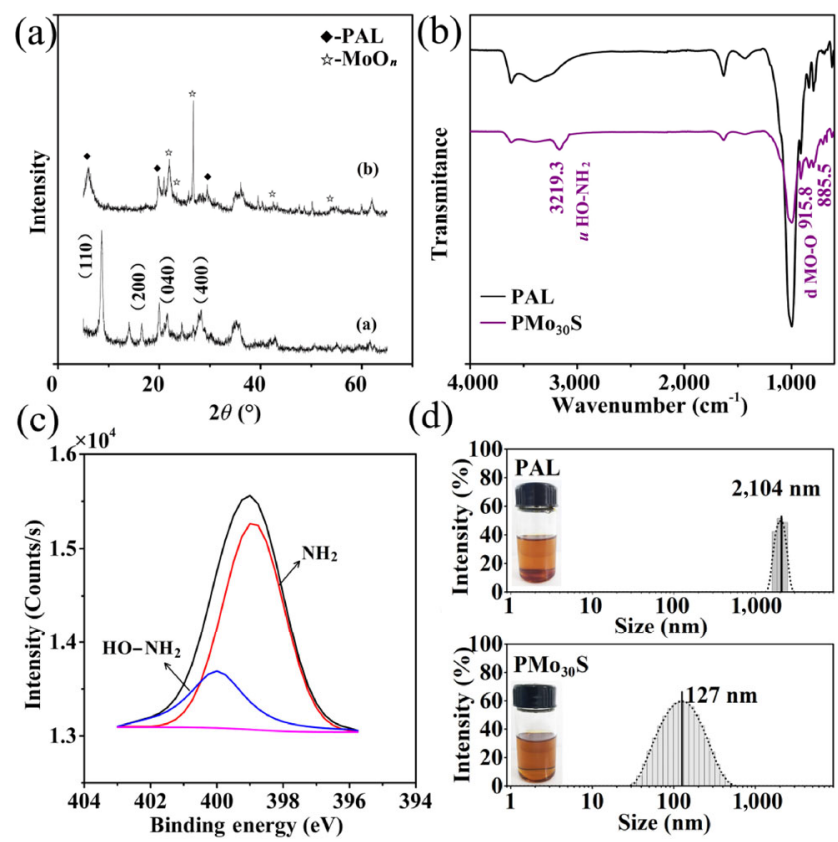

(d)

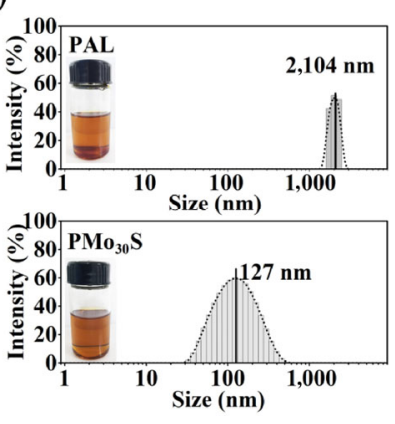

Fig. 1 Characteristics of the synthetic Amo-PMo nanoplatelets: (a) XRD spectra of synthetic Amo-PMo and PAL; (b) IR spectra of PAL and Amo-PMo; (c) HO- $\mathrm{NH}_{2}$ XPS spectra of the synthetic Amo-PMo nanoplatelets; (d) particle size of the unmodified PAL and modified Amo-PMo dispersed in the base oil after seven days. 
spectra was the peaks at 885.5 and $915.8 \mathrm{~cm}^{-1}$, which corresponds to the stretching vibration of the Mo-O groups. These values accurately match the characteristic peaks of Mo. As lubricant nano-additives for commercial applications, nanoplatelets should be well dispersed in the base oil. Here, the dispersion effect was investigated as $0.5 \mathrm{wt} \%$ additives were standing in the base oil for seven days. Considering that the unmodified PAL precipitated in the base oil (Fig. S2 (ESM)) and the modified Amo-PMo solution exhibited a transparent brown appearance, it can be speculated that the surface of the PAL was modified by APTES. To investigate the presence of APTES functional groups on the surface of nanoplatelets, we cleaned the Amo-PMo material three times with ethanol by sonication for $20 \mathrm{~min}$. Finally, APTES was shown to exist on the Amo-PMo surface by IR spectroscopy (Fig. 1(b)) and XPS (Fig. 1(c)). APTES underwent a hydrolysis reaction to generate active silanol intermediates $(\mathrm{Si}-\mathrm{OH})$, which interacted with the surface hydroxyl groups of the PAL, resulting in a hydrophobic $-\mathrm{NH}_{2}$ terminal group on the surface of the PAL. The $-\mathrm{NH}_{2}$ terminal group of APTES would be oriented toward the base oil. Therefore, the hydrophobic surface of the nanoplatelets allowed the additive to be better dispersed in the base oil. We performed a dynamic light scattering experiment to analyze the particle size of the additive in the base oil after seven days (Fig. 1(d)). It could be seen that the average particle size of the modified PAL was approximately $127 \mathrm{~m}$, whereas that of the unmodified PAL was 2,104 nm. We then observed that the lubricating fluid was still in a transparent state after 15 days (Fig. S2 (ESM)), which indicated that the synthetic additive could be well dispersed in the base oil within 15 days.

The SEM image in Fig. 2(a) shows that the nanoplatelets had a different morphology and more curled edges (Fig. S3 (ESM)) as compared to the conventional PAL. The main reason was that when the synthetic nanoplatelets were thinner, the edges were easily curled. The TEM image in Fig. 2(b) shows the morphology and dimensions of Amo-PMo. These nanomaterials were well dispersed in the solvent and uniformly spread on the substrate. The TEM image in Fig. S4 (ESM) displays the untreated PAL morphology. In general, the PAL materials agglomerated and adhered to each other, whereas Amo-PMo did not appear to agglomerate.

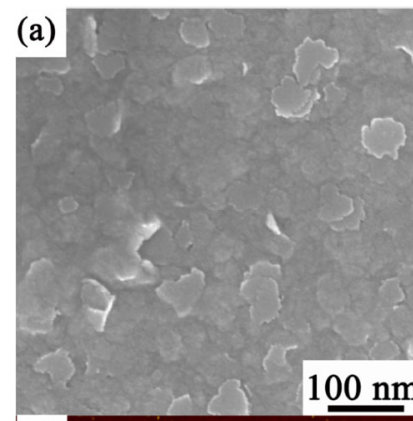

(b)

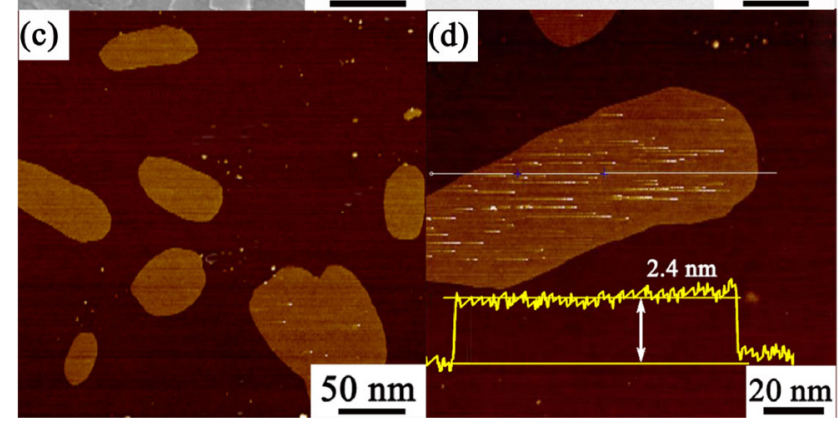

Fig. 2 Characteristics of the synthetic Amo-PMo nanoplatelets: (a) An SEM image of synthetic Amo-PMo; (b) a low magnification TEM image of synthetic Amo-PMo; (c) an AFM image of the synthetic Amo-PMo nanoplatelets; (d) cross-sectional profile of the synthetic Amo-PMo nanoplatelets.

APTES played a vital role in surface modification. To further confirm the thickness of the as-synthesized nanoplatelets, the samples were analyzed by AFM. The AFM image in Fig. 2(d) shows that the morphologies of the synthesized Amo-PMo nanomaterials were elliptical and their thicknesses were measured to be $2.4 \pm 0.2 \mathrm{~nm}[24]$.

\subsection{Tribological properties of the Amo-PMo nano- platelets}

In this study, the samples were labeled $\mathrm{PMo}_{n} \mathrm{~S}$ (with $n=$ 20,30 , and 50), where $n$ denotes the mass ratio of SN-Mo in the composite. To analyze the tribological properties of PAL, SN-Mo, and their synergistic effects, we tested 10 lubricants to determine if under the highpressure conditions, these additives could still reduce friction. The coefficient of friction (COF) of the upper against the bottom GCr15 balls at the normal load of $400 \mathrm{~N}$ at $75{ }^{\circ} \mathrm{C}$ is shown in Fig. 3. In the lubricating fluid, as the PAL content increased, the COF first decreased (0.50 wt $\%$ PAL) and then increased $(0.75 \mathrm{wt} \%$ PAL), as shown in Fig. 3(a). When the additive content was low, the layered structure of PAL could enter the 


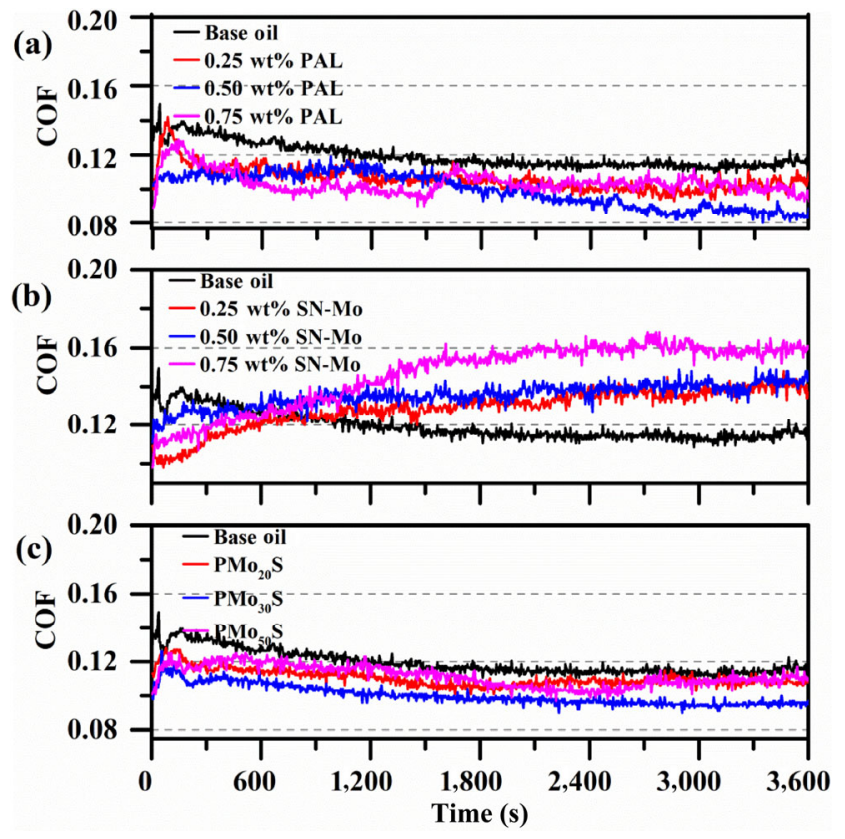

Fig. 3 COF curves of the samples: (a) for different PAL contents; (b) for different contents of SN-Mo; (c) for different contents of Amo-PMo.

contact areas between the two sliding surfaces. The interlayer sliding was relatively easy between the contact surfaces. However, when the additive content exceeded a certain amount, PAL was unable to enter the contact area, and the additive may be used as a three-body material, resulting in an increase in the COF [25]. The COF of the base oil with SN-Mo additive is shown in Fig. 3(b) and was much higher than that of the base oil. According to Hamrock-Dowson theory, the thickness of the lubricating film was 2.56 times of the surface roughness. Referring to the Streibeck curve, lubrication was known to be at the boundary of the mixed lubrication and elasto-hydrodynamic lubrication (EHL) regime, and as the viscosity increased (Fig. S6 (ESM)), the COF also increased [26]. After the Amo-PMo nanoplatelets were dispersed in the base oil, the COF obviously decreased. When the lubricating fluid between the sliding surfaces was the base oil, the COF tended to increase at the beginning of the tribological test. It was reasonable to speculate that the relatively high COF during this period was perhaps caused by much more severe contact stress of point contact geometry combined with the third-body effect in the running-in regime [27]. As time elapsed, the COF slowly decreased after the running-in stage.
When 0.5 wt $\%$ Amo-PMo was added, the COF tended to be lower and more stable than that of the base oil, and the COF was reduced by $15.99 \%$. Zinc dialkyldithophosphate (ZDDP) is commonly used as an organic lubricant additive, and its molecular formula is displayed in Fig. S7(c) (ESM). When ZDDP and graphene are used as lubricant additives, both will form a tribofilm on the sliding surfaces. The COF in these tests is shown in Fig. S7(a) (ESM).

To observe and compare the anti-wear properties of these samples, their wear scars were measured, and the results are shown in Fig. 4. The images of the wear scars were obtained using an optical microscope (Figs. 4(a)-4(d)), and the 3D contour topographies were obtained using a 3D white light interferometer (Figs. 4(e) and $4(\mathrm{f}))$. Figure $4(\mathrm{~g})$ shows the cross-section profile curve after the ball was worn. Figures 4(a)-4(d) show that when Amo-PMo was added to the base oil, the diameter of the wear scar became much smaller, and the wear scar showed no indication of furrows. Of the three samples with Amo-PMo, the ball tested with the $\mathrm{PMo}_{30} \mathrm{~S}$ sample had the smallest wear scar. In fact, the size of the wear scar had a substantial relationship with the synergistic effect (SN-Mo/PAL ratio) of Amo-PMo. When used as a nano-additive, the $\mathrm{PMo}_{50} \mathrm{~S}$ sample contained the most SN-Mo and was highly likely to scratch the sliding surfaces due to tumbling during the rubbing process [28].

The anti-wear properties of the 10 lubricant samples were evaluated to determine whether these additives could exhibit lower wear under high-pressure conditions. The wear scar diameter was measured using an optical microscope, and the wear volume was measured using a 3D white light interferometer. Figure 5(a) shows the wear of PAL. The wear conditions of PAL were ultimately worse than those of the base oil, indicating that PAL could enter the contact zone within a certain range. However, the adsorption performance was poor (Fig. S9(b) (ESM)), as PAL could slip out from the contact zone, which increased the wear of the sliding surface. As shown in Fig. 5(b), the wear condition of SN-Mo on the metal sliding surface was better than that of PAL. It was reasonable to speculate that SN-Mo had excellent adsorption properties and could adhere to the grooves on the contact area to protect the worn surface. After the Amo-PMo nanoplatelets were added to the base oil, 

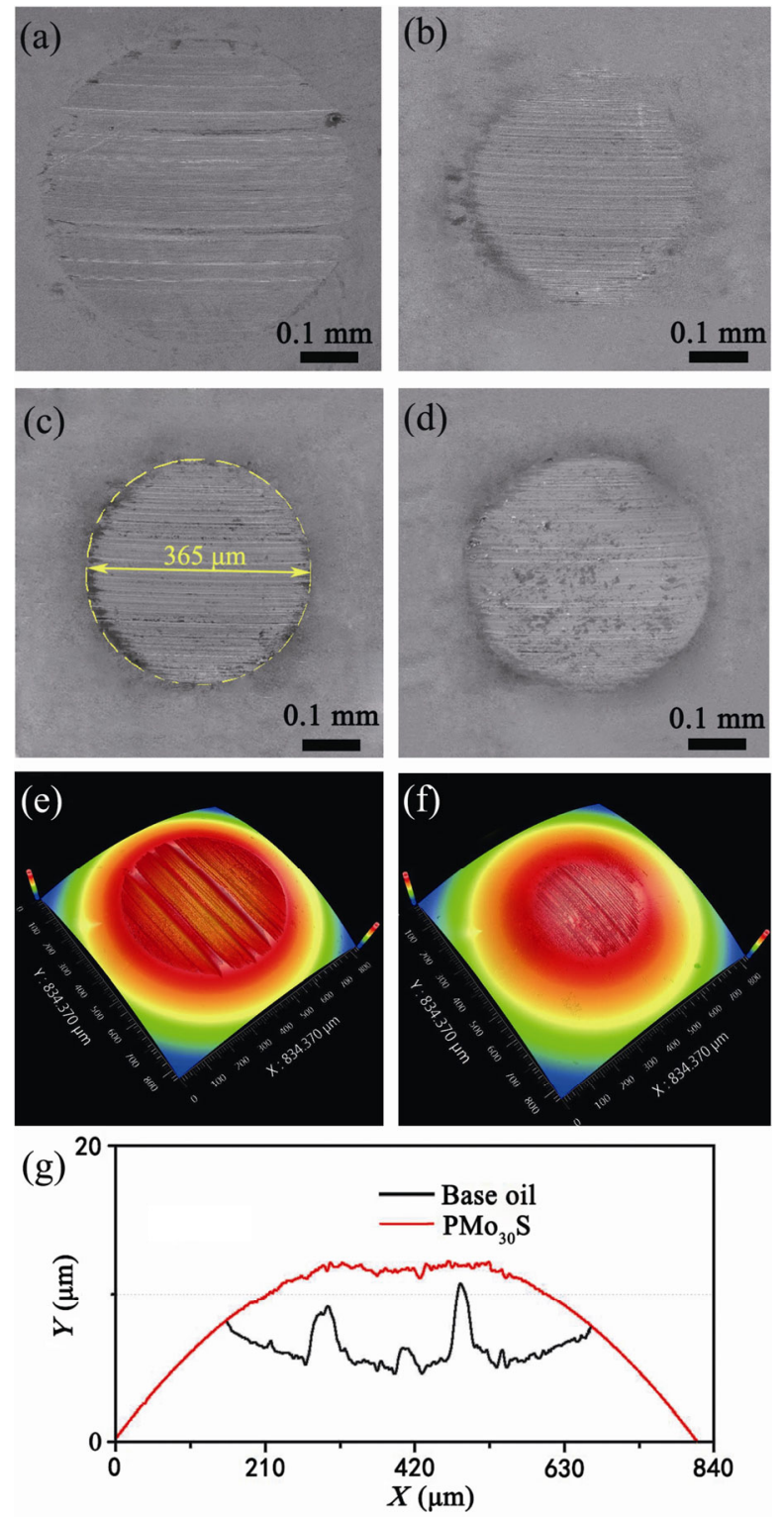

Fig. 4 Optical images of the wear scars tested with (a) the base oil sample, (b) the $\mathrm{PMo}_{20} \mathrm{~S}$ ball, (c) the $\mathrm{PMo}_{30} \mathrm{~S}$ ball, and (d) the $\mathrm{PMo}_{50} \mathrm{~S}$ ball. The 3D topography of the wear scars on the ball, which was lubricated with (e) the base oil and (f) $\mathrm{PMo}_{30} \mathrm{~S}$. (g) The line scans of the wear scars.

the anti-wear properties of the lubricating fluid were significantly improved (Fig. 5(c)). The nanoplatelet that improved the anti-wear properties of the lubricant most significantly was $\mathrm{PMo}_{30} \mathrm{~S}$, the wear scar diameter and wear volume of which were $63.3 \%$ and $49.6 \%$ of those of the base oil, respectively. The wear volumes of the $\mathrm{PMo}_{20} \mathrm{~S}$ and $\mathrm{PMo}_{50} \mathrm{~S}$ balls were $67,140.4$ and $80,976.1 \mu^{3}$, respectively, which were much smaller than that of the base oil $\left(1,244,705.4 \mu \mathrm{m}^{3}\right)$.
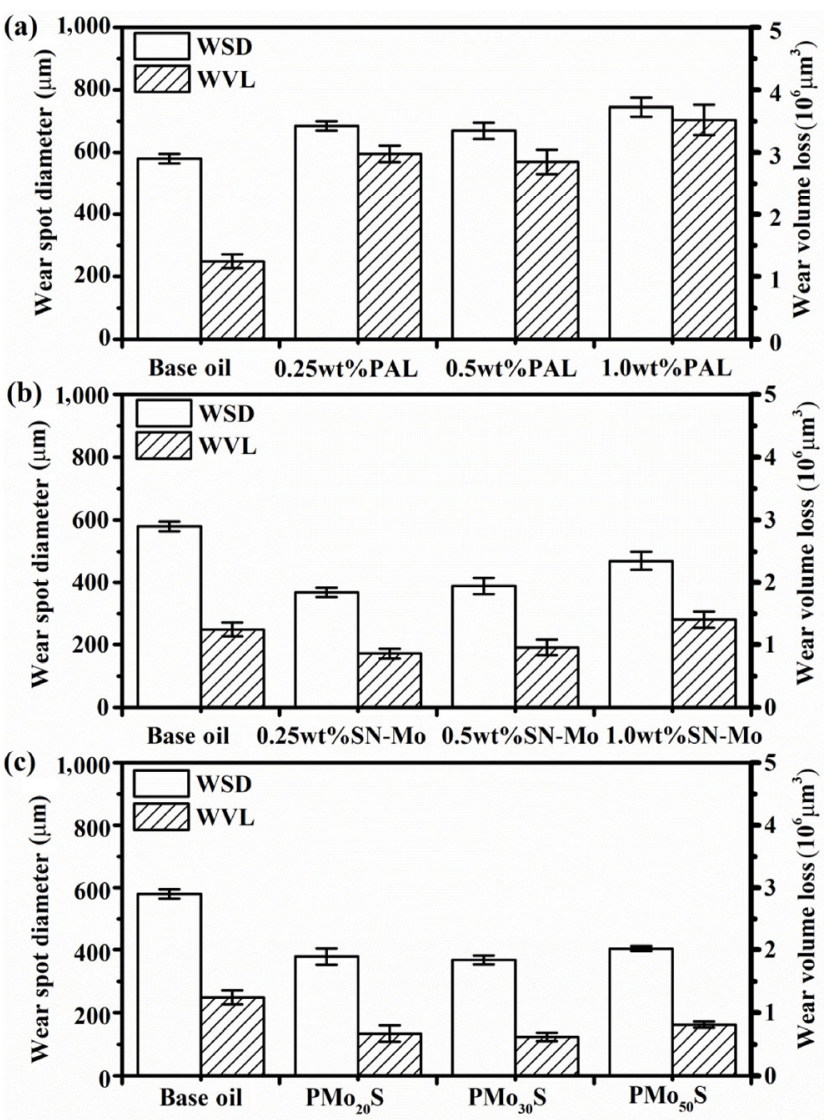

Fig. 5 Histograms of the wear spot diameter and wear volume loss rate for different lubricant contents: (a) PAL, (b) SN-Mo, and (c) Amo-PMo.

To compare the anti-wear properties of commercial additives, the anti-wear properties of ZDDP and graphene are shown in Fig. S7(b) (ESM).

\subsection{Wear scar morphology and elemental valence study}

The physical morphology and structural changes of the contact surface can directly affect the tribological behaviors of the sliding surfaces. Therefore, further investigating the bottom balls is necessary. First, after the tribological test, the balls tested with the base oil, $\mathrm{PAL}$, and $\mathrm{PMo}_{30} \mathrm{~S}$ were selected, and their wear scars were observed using SEM and EDS mapping analysis. The results are shown in Fig. 6. Compared with the base oil, the Amo-PMo additives formed a dark brown and relatively dense protective film on the wear scar of the sliding surfaces. Figure 6(a) shows the SEM image of the base oil sample. The deep, rough, and relatively wide furrows suggested that 

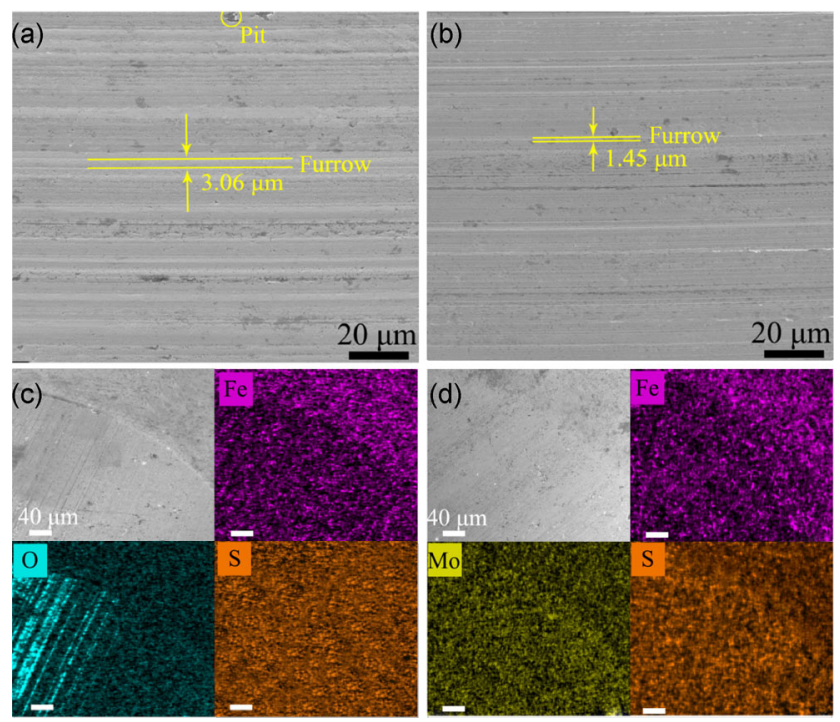

Fig. 6 SEM images of the surfaces after running for $1 \mathrm{~h}$ : (a) the base oil sample and (b) $\mathrm{PMo}_{30} \mathrm{~S}$ ball. EDS mapping analysis for the wear surface lubricated by (c) the base oil and (d) $0.5 \mathrm{wt} \%$ $\mathrm{PMo}_{30} \mathrm{~S}$.

the surface of the base oil sample ball was scraped by asperities and three-body particles. Figure 6(b) shows the surface image of the $\mathrm{PMo}_{30} \mathrm{~S}$ sample ball. The shallow and relatively narrow furrows indicated that the surface was clearly covered by tribofilm. Figure 6(c) shows the EDS mapping images of the ball tested with base oil. The oxygen element distribution on the worn surface was not very uniform, indicating that the protective tribofilm lubricated by base oil was not formed. As shown in Fig. S8 (ESM), thin and long layered abrasion debris was present on the contact surface. This was a typical delamination wear resulting from surface crack generation and propagation. When the rough peak of the contact surface slid on a relatively smooth surface, the smooth surface underwent a cyclic load, causing shear plastic deformation and accumulation on the surface layer, which caused periodic dislocations on the metal surface layer. During the tribological process, dislocation accumulated at a certain depth below the surface, and then cracks were formed. Finally, the material between the crack and surface was peeled off in the form of layered wear debris [29].

To investigate whether chemical reactions occur on the worn surface, we performed XPS characterization on the experimental sample, including that on the bearing steel coated with the lubricating fluid containing additive, and the other on the tribofilm after the tribological test. The result is shown in Fig. 7. The molybdenum element in the coating layer was only the valence state of the original $\mathrm{MoO}_{3}$ of the additive [30]. After the tribological test, a small peak of the molybdenum $(230.6 \mathrm{eV})$ appeared in the tribofilm, which corresponded to $\mathrm{MoS}_{2}$ of Mo $3 \mathrm{~d}_{5 / 2}$. Because the generated $\mathrm{MoS}_{2}$ was found in the tribofilm, no $\mathrm{MoS}_{2}$ component was present in the lubricating fluid. It was reasonable to speculate that the tribofilm was the product of a tribochemical reaction [31].

To understand the chemical composition of the tribofilm previously mentioned, we performed EDS mapping analysis and XPS characterization. Figure 8 illustrates the EDS mapping results of the sliding surfaces, which showed that the elements presented on the wear scar surface included $\mathrm{Mo}, \mathrm{Si}, \mathrm{Fe}, \mathrm{O}$, and $\mathrm{S}$. In addition, the Mo and $\mathrm{S}$ elements were uniformly distributed in the tribofilm, which indicated that Amo-PMo could be transferred to the contact surface by the tribochemical reaction. Figure S9 (ESM) shows EDS mapping images of the ball tested with the base oil and PAL samples. Compared with the Amo-PMo, the ball tested with PAL additives showed wider furrows on the sliding surfaces, and no $\mathrm{Mg}$ element distribution was present in the tribofilm, indicating that PAL could not be transferred to the worn surface. The oxygen element distribution on the worn surface was not very uniform, indicating that the protective tribofilm lubricated by PAL was not formed. XPS analysis was conducted to investigate the effects of the Amo-PMo additives on the elements on the worn surface and the valence changes to those elements. Note that, due to the accuracy limitations of XPS

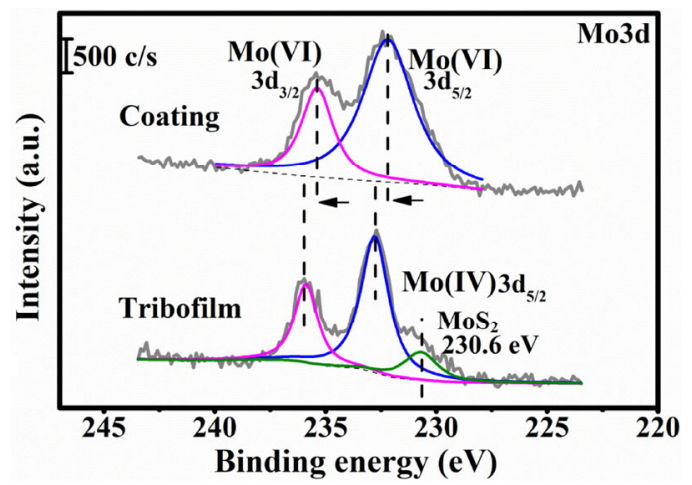

Fig. 7 XPS surface analysis of the samples: the coating and tribofilm. 

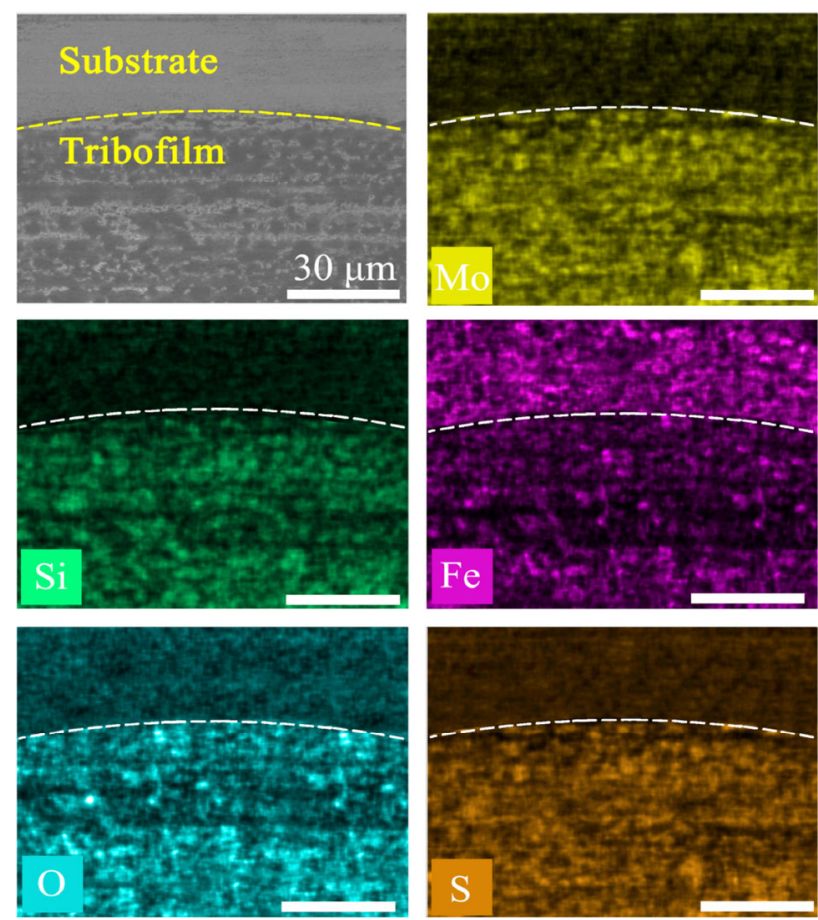

Fig. 8 EDS mapping analysis for the tribofilm on the wear scars.

devices, a large error occurred when the atomic mass fraction was less than $3 \%$. However, based on an analysis of the valence bond of an element and the composition of a compound, the XPS system was still very accurate. Figure 9 shows the elemental XPS spectra of the bottom balls of the $\mathrm{PMo}_{30} \mathrm{~S}$ sample after $\mathrm{Ar}^{+}$in situ sputtering. Figure 9(a) shows the XPS spectrum of Fe $2 p$, and the largest binding energy peak was assigned to the $\mathrm{Fe} 2 \mathrm{p}_{1 / 2}$ band $(710.5 \mathrm{eV})$, which also corresponds to the binding energy peak of $\mathrm{Fe}$ in $\mathrm{FeO}$. The Fe $2 p$ spectrum had a small peak at $720 \mathrm{eV}$, which (a)

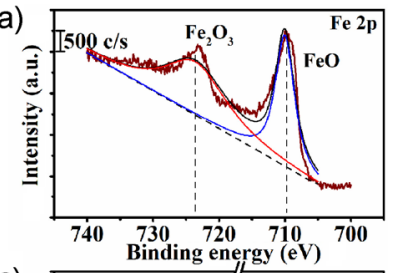

(c)

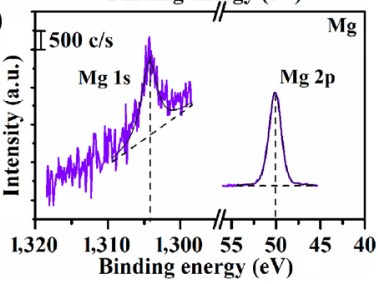

(b)

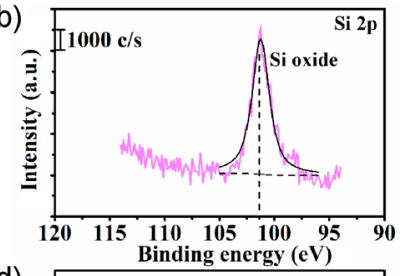

(d)

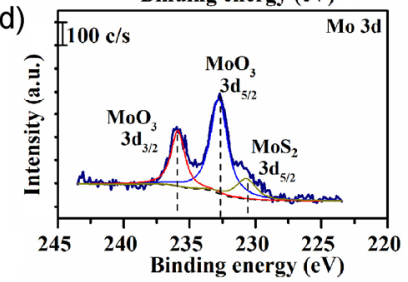

Fig. 9 XPS spectra of the elements on the surface of the $\mathrm{PMo}_{30} \mathrm{~S}$ sample: (a) Fe XPS spectrum, (b) Si XPS spectrum, (c) Mg XPS spectrum, and (d) Mo XPS spectrum. corresponds to the binding energy of monoatomic Fe and indicated that the content of monoatomic Fe in the tribofilm was low. Monoatomic Fe may have been present because of abrasive particles that fell off the asperities. The Fe $2 p$ XPS spectrum exhibited a high intensity peak at $723.5 \mathrm{eV}$, which corresponds to $\mathrm{Fe}_{2} \mathrm{O}_{3}$ of Fe $2 p_{3 / 2}$ [32]. Figure S11(a) (ESM) shows the XPS spectrum of $C 1 \mathrm{~s}$, in which the valence state of $C 1 \mathrm{~s}$ could be divided into two peaks corresponding to the two types of periodic structure. It could be observed that the $\mathrm{C} 1 \mathrm{~s}$ largest binding energy peak was $\mathrm{C}-\mathrm{C}$ $(284.8 \mathrm{eV})$, which also corresponded to the binding energy peak of the base oil. A small peak was observed at $284.6 \mathrm{eV}$, which was a C-O [33]. Figure S11(b) (ESM) shows the XPS spectrum of $\mathrm{O} 1 \mathrm{~s}$ with a peak binding energy of $531.80 \mathrm{eV}$ and an accompanying peak at $529.37 \mathrm{eV}$. The peak corresponding to $\mathrm{O} 1 \mathrm{~s}$ was very wide, which indicated that $\mathrm{O}$ had multiple valences and O existed mainly as silicates. Under the thermochemical reactions, an oxidation reaction occurred on the sliding surface. The newly formed surface of the wear scar reacted with the zeolite in the PAL material and resulted in the formation of $\mathrm{Fe}_{2} \mathrm{O}_{3}$ [34]. The XPS spectrum of Si 2 p shown in Fig. 9(b) exhibits a peak binding energy at $103.49 \mathrm{eV}$, indicating that $\mathrm{Si}$ was present in the form of $\mathrm{SiO}_{2}$ and silicates. Figure 9(c) shows the XPS spectra of $\mathrm{Mg} 1 \mathrm{~s}$ and $\mathrm{Mg} 2 \mathrm{p}$, and the peak binding energy were at 1303.9 and $50.1 \mathrm{eV}$, respectively, indicating that $\mathrm{Mg}$ existed in the form of $\mathrm{MgO}$. Figure 9(d) shows the XPS spectrum of Mo 3d, in which the peak binding energy was at $231.1 \mathrm{eV}$, corresponding to $\mathrm{Mo} 3 \mathrm{~d}_{5 / 2}$ of $\mathrm{MoO}_{3}$. The Mo element in the tribofilm exhibited a small peak of $230.6 \mathrm{eV}$, which corresponds to $\mathrm{MoS}_{2}$ of $\mathrm{Mo} 3 \mathrm{~d}_{5 / 2}$.

\subsection{Film thickness calculation}

According to the Hamrock-Dowson theory of point contacts, the minimum film thickness $\left(h_{\min }\right)$ can be evaluated by the following equation [35]:

$$
h_{\min }=3.63 \frac{U^{0.68} G^{0.49}}{W^{0.073}}\left(1-\mathrm{e}^{-0.68 k}\right) R
$$

where $\lambda=h_{\min } / \sigma, U=\eta v / E R, G=\alpha E$, and $W=F / E R^{2}$. Here, $\lambda$ is the ratio used to determine the lubrication method of the contact area. When $\lambda$ is less than 1 , the lubrication regime is a boundary lubrication, but 
when $\lambda$ ranges from 1 to 3 , mixed lubrication occurs; if $\lambda$ is greater than 3 , lubrication occurs by EHL or EHD [36-38]. In addition, $\sigma$ is the roughness of the two sliding surfaces $(\sim 0.21 \mu \mathrm{m})$, and $\eta$ is the dynamic viscosity of the lubricating fluid. At the beginning of the test, the base oil had a dynamic viscosity of $29.5 \mathrm{cP}$ at room temperature. After the tribological test, the base oil had a dynamic viscosity of $17.5 \mathrm{cP}$ at $75^{\circ} \mathrm{C}$. The dynamic viscosity of the lubricating fluid was tested at shear rates from 10 to 1,000 s-1 (Fig. S6 (ESM)). During the test of each dynamic viscosity, the viscosity did not change significantly with the shear rate, indicating that the lubricating fluid was a Newtonian fluid. Finally, $v(\sim 1.6 \mathrm{~m} / \mathrm{s})$ is the average tangent speed of the outer edge of the ball, $E$ is the effective elastic modulus, $\alpha\left(1.5 \times 10^{-9} \mathrm{~Pa}^{-1}\right)$ is the viscosity-pressure coefficient [39], $R(\sim 12.7 \mathrm{~mm})$ is the radius of the ball, $F(400 \mathrm{~N})$ is the load applied by the main shaft, and $k(\sim 1)$ is the ellipticity of the ball [40]. $\lambda$ was calculated to be approximately 0.9688 , and it can be concluded that the contact zone in the initial stage was in the boundary lubrication state, which indicated that the initial wear was severe.

Following the friction test, a circular flat worn surface having a diameter of approximately $396 \mu \mathrm{m}$ was produced, and thus, the Hamrock-Dowson film thickness formula was not suitable. However, in EHL theory, the deformation of the ball followed Hertzian contact theory, and an approximate method could be proposed to estimate the film thickness at the final stage, which is expressed by the following equation [41]:

$$
R^{*}=\frac{2 E\left(r^{*}\right)^{3}}{3 F R}
$$

where $r^{*}$ is the radius of the worn circular flat surface of the bottom ball, $R$ is the radius of the bottom ball, and $F$ is the load applied by the main shaft. According to the Hertzian contact theory, for the test of the $\mathrm{PMo}_{30} \mathrm{~S}$ additive, Eq. (2) is substituted into Eq. (1). It can be concluded that a small amount of Amo-PMo hardly affects the oil film thickness. $\lambda$ is approximately 2.56, from which it can be inferred that the lubrication of the contact zone in the final stage occurs by mixed lubrication.

As reported in Refs. [42, 43], the Young's modulus and ultimate strength of monolayer nanoplatelets were much higher than those of steel. Klemenz et al. [44] provided evidence that as long as the $2 \mathrm{D}$ material was not destroyed, the 2D material could be an excellent coating with low friction and wear. According to the analysis, the ability of $2 \mathrm{D}$ material nano-additives to enter the contact area is a major concern. Fortunately, the synthesized Amo-PMo nanoplatelets have this ability. First, APTES modifies the surface of the PAL to make them float in the base oil without agglomeration. Second, thinner nanoplatelets can enter the contact area. In our study, as shown in Fig. 10(b), when micro peaks were moving toward each other, the nanoplatelets could enter the contact area. When two rough peaks were in contact, because of the presence of the nanoplatelets between them, they may not have contacted each other directly. After the two peaks separated, the nanoplatelets may have adhered to one of the peaks or returned (floated) into the lubricant. In addition, SN-Mo chemically reacted with $S_{x}$ and adhered to the worn surface, thus reducing the amount of wear. Numerous micropeaks of the sliding surface were making contact and separating, so this process occurred numerous times. As long as Amo-PMo could enter the contact area, the sliding surface exhibited excellent anti-wear property. Regarding ordinary PAL, they were more easily pushed away due to their larger size, resulting in wear under high loads.

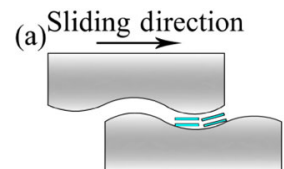

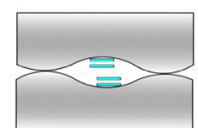

Indirect contact with shearing

(b)

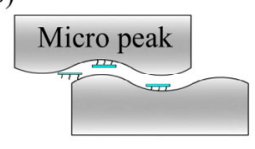

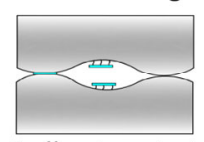

Indirect contact with deformation ऍPAL

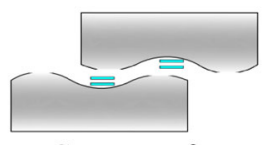

Contact surface with wear

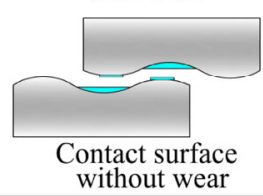

PAL $\leadsto$ Amo-PMo
Fig. 10 Schematics of the proposed lubrication mechanism of (a) PAL and (b) Amo-PMo nanoplatelets.

\section{Conclusions}

New type of molybdenum-dotted PAL nanoplatelets were synthesized using the AMOST method. The as-synthesized samples, their morphologies and physicochemical characteristics were analyzed by 
TEM, SEM, AFM, XPS, and DLS. The synthesized nanoplatelets were of only a few layers. Compared with synthetic additives and commercial additives, it was found that the Amo-PMo possessed superior ability to increase the tribological properties of the lubricating fluid. Finally, these additives reduced the COF and wear scar diameter by $16 \%$ and $63 \%$, respectively, compared to those under the base oil lubrication. Among these additives, the $0.5 \mathrm{wt} \% \mathrm{PMo}_{30} \mathrm{~S}$ lubricant additive exhibited excellent tribological properties and formed a more protective tribofilm than did the other additives during the tests.

\section{Acknowledgements}

This project was supported by Major Science and Technology Project in Guizhou Province (Grant No. Q.K.H.Z.D.Z.X.Z[2019]3016), National Natural Science Foundation of China (Grant Nos. 51527901, 51875303, 51905294, and 51465008), Science and Technology Innovation Team Project in Guizhou Province (Grant No. Q.K.H.P.T.R.C[2020]5020), Preferred Project of Scientific and Technological Activities for Personnel Studying Abroad in Guizhou Province (Grant No. Q.R.X.M.Z.Z.H. T(2018)0001), Science and Technology Planning Project in Guizhou Province (Grant No. Q.K.H.P.T.R. C[2017]5788), and Training Plan for High-level Innovative Talent in Guizhou Province (Grant No. Q.K.H.P.T.R.C[2016]5659).

Open Access This article is licensed under a Creative Commons Attribution 4.0 International License, which permits use, sharing, adaptation, distribution and reproduction in any medium or format, as long as you give appropriate credit to the original author(s) and the source, provide a link to the Creative Commons licence, and indicate if changes were made.

The images or other third party material in this article are included in the article's Creative Commons licence, unless indicated otherwise in a credit line to the material. If material is not included in the article's Creative Commons licence and your intended use is not permitted by statutory regulation or exceeds the permitted use, you will need to obtain permission directly from the copyright holder.

To view a copy of this licence, visit http://creativecommons.org/licenses/by/4.0/.
Electronic Supplementary Material: Supplementary material is available in the online version of this article at https://doi.org/10.1007/s40544-019-0347-6.

\section{References}

[1] Kumar P, Wani M F. Tribological characterisation of graphene oxide as lubricant additive on hypereutectic $\mathrm{Al}-25 \mathrm{Si} /$ Steel tribopair. Tribol Trans 61(2): 335-346 (2018)

[2] Nian J Y, Chen L W, Guo Z G, Liu W M. Computational investigation of the lubrication behaviors of dioxides and disulfides of molybdenum and tungsten in vacuum. Friction 5(1): 23-31 (2017)

[3] Uflyand I E, Zhinzhilo V A, Burlakova V E. Metalcontaining nanomaterials as lubricant additives: State-of-theart and future development. Friction 7(2): 93-116 (2019)

[4] Greco A, Mistry K, Sista V, Eryilmaz O, Erdemir A. Friction and wear behaviour of boron based surface treatment and nano-particle lubricant additives for wind turbine gearbox applications. Wear 271(9-10): 1754-1760 (2011)

[5] Silva A F T, Burggraeve A, Denon Q, Van der Meeren P, Sandler N, Van Den Kerkhof T, Hellings M, Vervaet C, Remon J P, Lopes J A, et al. Particle sizing measurements in pharmaceutical applications: Comparison of in-process methods versus off-line methods. Eur J Pharm Biopharm 85(3): 1006-1018 (2013)

[6] Middea A, Fernandes T L A P, Neumann R, Gomes O D F M, Spinelli L S. Evaluation of $\mathrm{Fe}(\mathrm{III})$ adsorption onto palygorskite surfaces. Appl Surf Sci 282: 253-258 (2013)

[7] Kong Y, Ge H L, Xiong J X, Zuo S X, Wei Y, Yao C, Deng L H. Palygorskite polypyrrole nanocomposite: A new platform for electrically tunable drug delivery. Appl Clay Sci 99: 119-124 (2014)

[8] Chen M D, Jiang W, Wang F H, Shen P, Ma P C, Gu J J, Mao J Y, Li F S. Synthesis of highly hydrophobic floating magnetic polymer nanocomposites for the removal of oils from water surface. Appl Surf Sci 286: 249-256 (2013)

[9] Li X Z, Zhang Z S, Chao Y, Lu X W, Zhao X B, Ni C Y. Attapulgite- $\mathrm{CeO}_{2} / \mathrm{MoS}_{2}$ ternary nanocomposite for photocatalytic oxidative desulfurization. Appl Surf Sci 364: 589-596 (2016)

[10] Lee J B. Effects of alloying elements, Cr, Mo and $\mathrm{N}$ on repassivation characteristics of stainless steels using the abrading electrode technique. Mater Chem Phys 99(2-3): 224-234 (2006)

[11] Wang Q, O'Hare D. Large-scale synthesis of highly dispersed layered double hydroxide powders containing delaminated single layer nanosheets. Chem Commun 49(56): 6301-6303 (2013)

[12] Zhang B S, Xu B S, Xu Y, Ba Z X, Wang Z Z. Lanthanum effect on the tribological behaviors of natural serpentine as lubricant additive. Tribol Trans 56(3): 417-427 (2013) 
[13] Qi X W, Jia Z N, Chen H M, Yang Y L, Wu Z. Self- repairing characteristics of serpentine mineral powder as an additive on steel-chromium plating pair under high temperature. Tribol Trans 56(3): 516-520 (2013)

[14] Yue W, Wang C B, Liu Y D, Huang H P, Wen Q F, Liu J J. Study of the regenerated layer on the worn surface of a cylinder liner lubricated by a novel silicate additive in lubricating oil. Tribol Trans 53(2): 288-295 (2010)

[15] Wang H D, Liu Y H, Liu W R, Wang R, Wen J G, Sheng H P, Peng J F, Erdemir A, Luo J B. Tribological behavior of NiAl-Layered double hydroxide nanoplatelets as oil-based lubricant additives. ACS Appl Mater Interfaces 9(36): 3089130899 (2017)

[16] Abdo J, Haneef M D. Clay nanoparticles modified drilling fluids for drilling of deep hydrocarbon wells. Appl Clay Sci 86: 76-82 (2013)

[17] Lai S Q, Li T S, Liu X J, Lv R G, Yue L. The tribological properties of PTFE filled with thermally treated nanoattapulgite. Tribol Int 39(6): 541-547 (2006)

[18] Lai S Q, Li T S, Liu X J, Lv R G. A study on the friction and wear behavior of PTFE filled with acid treated nanoattapulgite. Macromol Mater Eng 289(10): 916-922 (2004)

[19] Nan F, Xu Y, Xu B S, Gao F, Wu Y X, Li Z G. Effect of Cu nanoparticles on the tribological performance of attapulgite base grease. Tribol Trans 58(6): 1031-1038 (2015)

[20] Feng N, Xu Y, Xu B S, Gao F, Wu Y X, Li Z G. Tribological performance of attapulgite Nano-fiber/Spherical Nano-Ni as lubricant additive. Tribol Lett 56(3): 531-541 (2014)

[21] Tian G Y, Wang W B, Zong L, Kang Y R, Wang A Q. From spent dye-loaded palygorskite to a multifunctional palygorskite/ carbon/Ag nanocomposite. RSC Adv 6(48): 41696-41706 (2016)

[22] Zhong L F, Tang A D, Wen X, Yan P, Wang J J, Lin T, Chen J. New finding on $\mathrm{Sb}(2-3 \mathrm{~nm})$ nanoparticles and carbon simultaneous anchored on the porous palygorskite with enhanced catalytic activity. J Alloys Compd 743: 394402 (2018)

[23] Wang J Y, Huang L, Gao Y S, Yang R Y, Zhang Z, Guo Z H, Wang Q. A simple and reliable method for determining the delamination degree of nitrate and glycine intercalated LDHs in formamide. Chem Commun 50(70): 10130-10132 (2014)

[24] Xu Z P, Stevenson G, Lu C Q, Lu G Q. Dispersion and size control of layered double hydroxide nanoparticles in aqueous solutions. J Phys Chem B 110(34): 16923-16929 (2006)

[25] Lakshminarayana G, Qiu J R. Photoluminescence of $\operatorname{Pr}^{3+}$, $\mathrm{Sm}^{3+}$ and $\mathrm{Dy}^{3+}$-doped $\mathrm{SiO}_{2}-\mathrm{Al}_{2} \mathrm{O}_{3}-\mathrm{BaF}_{2}-\mathrm{GdF}_{3}$ glasses. $J$ Alloys Compd 476(1-2): 470-476 (2009)

[26] Jakiela R, Gas K, Sawicki M, Barcz A. Diffusion of Mn in gallium nitride: Experiment and modelling. J Alloys Compd 771: 215-220 (2019)

[27] Zou R B, Liu F F, Wang A L, Pan L C, Wang Z L. Lasing mechanism of $\mathrm{ZnO}$ nanowires/nanobelts at room temperature. J Phys Chem B 110(26): 12865-12873 (2006)

[28] Stachowiak G W. Particle angularity and its relationship to abrasive and erosive wear. Wear 241(2): 214-219 (2000)

[29] Grippaudo C, Cancellieri D, Grecolini M E, Deli R. Comparison between different interdental stripping methods and evaluation of abrasive strips: SEM analysis. Prog Orthod 11(2): 127-137 (2010)

[30] Martin J M, Le Mogne T, Grossiord C, Palermo T. Tribochemistry of ZDDP and MoDDP chemisorbed films. Tribol Lett 2(3): 313-326 (1996)

[31] Baker M A, Gilmore R, Lenardi C, Gissler W. XPS investigation of preferential sputtering of $\mathrm{S}$ from $\mathrm{MoS}_{2}$ and determination of $\mathrm{MoS}_{x}$ stoichiometry from Mo and S peak positions. Appl Surf Sci 150(1-4): 255-262 (1999)

[32] Meyer E. Physics. Controlling friction atom by atom. Science 348(6239): 1089 (2015)

[33] Lahiri J, Lin Y, Bozkurt P, Oleynik I I, Batzill M. An extended defect in graphene as a metallic wire. Nat Nanotechnol 5(5): 326-329 (2010)

[34] Moncoffre N, Hollinger G, Jaffrezic H, Marest G, Tousset J. Temperature influence during nitrogen implantation into steel. Nucl Instrum Meth Phys Res B 7-8: 177-183 (1985)

[35] Hamrock B J, Dowson D. Discussion: "Isothermal elastohydrodynamic lubrication of point contacts: Part IIIfully flooded results". J Lubr Technol 99: 275-276 (1977)

[36] Dowson D. Tribological principles in metal-on-metal hip joint design. Proc Inst Mech Eng H 220(2): 161-171 (2006)

[37] Etsion I. Modeling of surface texturing in hydrodynamic lubrication. Friction 1(3): 195-209 (2013)

[38] Luo J B, Lu X C, Wen S Z. Developments and unsolved problems in nano-lubrication. Progr Nat Sci 11(3): 173-183 (2001)

[39] Horne R A, Johnson D S. The viscosity of water under pressure. J Phys Chem 70(7): 2182-2190 (1966)

[40] Mia S, Ohno N. Prediction of pressure-viscosity coefficient of lubricating oils based on sound velocity. Lubr Sci 21(9): 343-354 (2009)

[41] Xie G X, Luo J B, Liu S H, Guo D, Zhang C H. "Freezing" of nanoconfined fluids under an electric field. Langmuir 26(3): 1445-1448 (2010)

[42] Bertolazzi S, Brivio J, Kis A. Stretching and breaking of ultrathin $\mathrm{MoS}_{2}$. ACS Nano 5(12): 9703-9709 (2011)

[43] Tu Q, Spanopoulos I, Yasaei P, Stoumpos C C, Kanatzidis M G, Shekhawat G S, Dravid V P. Stretching and breaking of ultrathin 2D hybrid organic-inorganic perovskites. ACS Nano 12(10): 10347-10354 (2018)

[44] Klemenz A, Pastewka L, Balakrishna S G, Caron A, Bennewitz R, Moseler M. Atomic scale mechanisms of friction reduction and wear protection by graphene. Nano Lett 14(12): 7145-7152 (2014) 


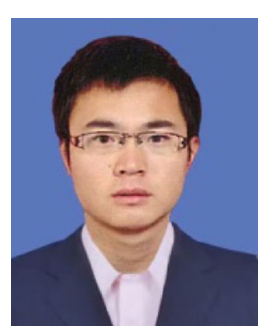

Kunpeng WANG. He obtained his B.S. degree in mechanical engineering from Guizhou University, China. After then, he is a Ph.D. student in

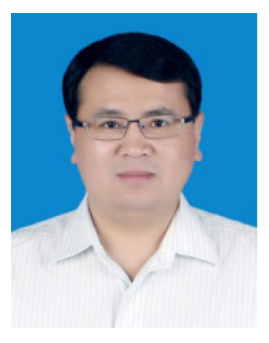

Huaichao WU. He obtained his Ph.D. degree in 2008 from State Key Laboratory of Fluid Transmission and Control, Zhejiang University, China. From April 2013 to April 2015, he carried out postdoctoral research in State key Laboratory of

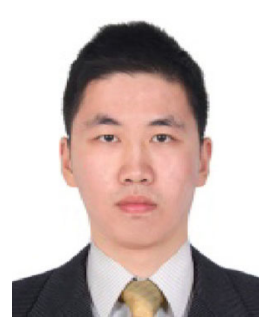

Hongdong WANG. He got his Ph.D. from State Key Laboratory of Tribology at Tsinghua University in 2018 and now is a postdoctor at

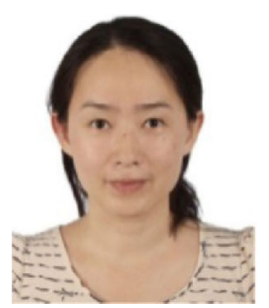

Yuhong LIU. She received her Ph.D. degree in Chinese Academy of Sciences Key Laboratory of Molecular Nanostructure \& Nanotechnology Institute of Chemistry, CAS, Beijing, China, in 2005. She is

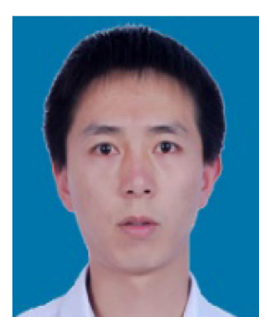

Lv YANG. He received his Ph.D. degree in 2012 from Guizhou University, China. He is currently an associate professor and master's

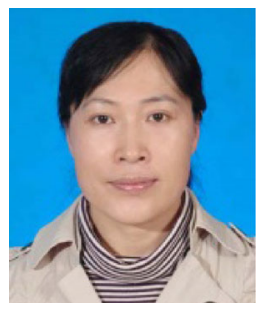

mechanical engineering in Guizhou University. His research interests are interfacial sliding properties and applications of two-dimensional layered materials as lubricant additives.

Tribology at Tsinghua University, China. He was invited to visit University of California, Davis, USA as a visiting scholar for one year in May 2016. He is now working as a professor in School of Mechanical Engineering, Guizhou University, China. He has been engaged in the research of friction, lubrication, and electrohydraulic control technology.

Tsinghua University, China. His research topics focus on the water-based superlubricity and the application of two dimensional nanomaterials in tribology.

an associate professor at the State Key Laboratory of Tribology of Tsinghua University, China, from 2005. Her research areas cover nanotribology, nanostructure, nanotechnology of surface and interface, chemicalmechanical planarization, and water-based lubrication.

supervisor in School of Mechanical Engineering, Guizhou University. His research areas cover lubricating oil/grease, nanomaterials, tribology, and surface engineering.

supervisor in School of Mechanical Engineering, Guizhou University. Her research interests include friction, lubrication, and mechatronic engineering. 